\title{
Is There Evidence of Cost Benefits of Electronic Medical Records, Standards, or Interoperability in Hospital Information Systems? Overview of Systematic Reviews
}

Zilma Silveira Nogueira Reis ${ }^{1 *}, \mathrm{MD}, \mathrm{PhD}$; Thais Abreu Maia ${ }^{2^{*}}, \mathrm{MBA}, \mathrm{MSc}$; Milena Soriano Marcolino ${ }^{3 *}, \mathrm{MD}, \mathrm{PhD}$; Francisco Becerra-Posada ${ }^{4 *}$, MD, DrPH; David Novillo-Ortiz ${ }^{4 *}$, MLIS, PhD; Antonio Luiz Pinho Ribeiro ${ }^{5 *}$, MD, PhD

${ }_{1}^{1}$ Informatics Center in Health, Obstetrics and Gynecology Department, Universidade Federal de Minas Gerais, Belo Horizonte, Minas Gerais, Brazil

${ }^{2}$ State Health Secretariat of Minas Gerais, Planning Advisory, Belo Horizonte, Minas Gerais, Brazil

${ }^{3}$ Faculty of Medicine, Internal Medicine Department, Universidade Federal de Minas Gerais, Belo Horizonte, Minas Gerais, Brazil

${ }^{4}$ Pan American Health Organization, Washington, DC, DC, United States

${ }^{5}$ Medical School, Telehealth Center, Hospital das Clínicas, Internal Medicine Department, Universidade Federal de Minas Gerais, Belo Horizonte, Minas Gerais, Brazil

*all authors contributed equally

\section{Corresponding Author:}

Zilma Silveira Nogueira Reis, MD, PhD

Informatics Center in Health

Obstetrics and Gynecology Department

Universidade Federal de Minas Gerais

Av. Prof. Alfredo Balena, 190

Belo Horizonte, Minas Gerais, 30130100

Brazil

Phone: 553134099648

Email: zilma.medicina@gmail.com

\begin{abstract}
Background: Electronic health (eHealth) interventions may improve the quality of care by providing timely, accessible information about one patient or an entire population. Electronic patient care information forms the nucleus of computerized health information systems. However, interoperability among systems depends on the adoption of information standards. Additionally, investing in technology systems requires cost-effectiveness studies to ensure the sustainability of processes for stakeholders.
\end{abstract}

Objective: The objective of this study was to assess cost-effectiveness of the use of electronically available inpatient data systems, health information exchange, or standards to support interoperability among systems.

Methods: An overview of systematic reviews was conducted, assessing the MEDLINE, Cochrane Library, LILACS, and IEEE Library databases to identify relevant studies published through February 2016. The search was supplemented by citations from the selected papers. The primary outcome sought the cost-effectiveness, and the secondary outcome was the impact on quality of care. Independent reviewers selected studies, and disagreement was resolved by consensus. The quality of the included studies was evaluated using a measurement tool to assess systematic reviews (AMSTAR).

Results: The primary search identified 286 papers, and two papers were manually included. A total of 211 were systematic reviews. From the 20 studies that were selected after screening the title and abstract, 14 were deemed ineligible, and six met the inclusion criteria. The interventions did not show a measurable effect on cost-effectiveness. Despite the limited number of studies, the heterogeneity of electronic systems reported, and the types of intervention in hospital routines, it was possible to identify some preliminary benefits in quality of care. Hospital information systems, along with information sharing, had the potential to improve clinical practice by reducing staff errors or incidents, improving automated harm detection, monitoring infections more effectively, and enhancing the continuity of care during physician handoffs.

Conclusions: This review identified some benefits in the quality of care but did not provide evidence that the implementation of eHealth interventions had a measurable impact on cost-effectiveness in hospital settings. However, further evidence is needed to infer the impact of standards adoption or interoperability in cost benefits of health care; this in turn requires further research. 
(JMIR Med Inform 2017;5(3):e26) doi: 10.2196/medinform.7400

\section{KEYWORDS}

electronic medical records; standards; medical information exchange; health information exchange; cost; benefits and costs

\section{Introduction}

Information technology (IT) applied to health care, or electronic health (eHealth) [1], ostensibly offers numerous benefits to the quality of health information, particularly in its recording, retrieval, and use. Patients can benefit directly from safe and accessible electronic clinical information for better decision making [2]. However, demographics and patient data are highly fragmented and distributed across multiple unintegrated systems [3]. Comprehensive and consistent health care, leading to effective use of services, requires the computerization of health data for more efficient communication. To achieve this, standardized information channels are needed to make syntactic interoperability possible among electronic records systems. Semantic interoperability is necessary to guarantee the consistency of information, as health information models require adopting standards to support communication [2]. Even if the standardization of electronic health records (EHRs) in eHealth systems is accomplished, health data sharing will continue to be a global challenge. Few publications exist concerning the impact of medical records and interoperability among health systems in cost and benefits of patient care.

Improvements in health and economic indicators are relevant metrics to justify IT investments. Indeed, planning and investing in IT is necessary for the efficient use of information that not only advances health care but also holds financial, social, cultural, and ethical benefits. Comparative cost-effectiveness studies guide agencies and institutions in choosing the best option for desired clinical outcomes and costs, which is the key to ensuring the sustainability of government health systems and their welfare programs $[3,4]$.

This review analyzes systematic reviews addressing the cost benefit and effectiveness of electronic medical records (EMR), standards adoption, or interoperability to discuss the benefits, drawbacks, and lessons learned from the implementation of actions related to eHealth and serves as a reference for government representatives and stakeholders. The assessment of the involvement of government and private health institutions in the implementation and maintenance of eHealth interventions that were tested and valuated worldwide is also of interest. The study was directed by 2 questions: What evidence exists regarding the impact of computerizing applications, standards, health information exchange, or interoperability to support the quality of care or patient outcomes in hospital settings? What critical cost-benefit evidence is published to provide a clear understanding of the value of eHealth implementations?

\section{Methods}

\section{Basic Concepts and International Standards On eHealth}

The terms used here to describe eHealth technologies are available in Multimedia Appendix 1. Despite different meanings, some papers use the terms electronic medical record (EMR) and electronic health record (EHR) synonymously. A relevant dissimilarity exists between health information exchange for systems integration and interoperability. The former refers to organizational framework for the dissemination of electronic health care information or clinical data across health-related institutions and systems to enhance patient care [5]. The latter relates to the the ability or capability of two or more systems to exchange information and use the exchanged information, which may support a longitudinal record widely available across institutions and over life spans [6]. Additionally, in a more specific context, "interoperability means the ability of health information systems to work together within and across organizational boundaries in order to advance the effective delivery of health care for individuals and communities" [7].

It is also important to emphasize that interoperability is usually divided into (1) syntactic interoperability: the capability of two or more systems to communicate and exchange data through specified data formats and communication protocols, and (2) semantic interoperability: the ability for data shared by systems to be understood at the level of fully defined domain concepts [8].

Worldwide coordinated efforts resulted in the development of standards to define an EHR as one or more repositories of actionable information by computers. The European Committee for Standardization (CEN), health level seven (HL7), International Organization for Standardization (ISO), and openEHR Foundation are nonprofit organizations dedicated to providing frameworks and standards. Terminologies, EHR specifications, and information models are proposed by these international standards organizations that support the exchange, integration, interoperability, and retrieval of electronic health information [6].

To better represent the meaning of standards in the primary selected systematic reviews, we adopted the generic definition for the term as: "A document adopted by consensus by a recognized entity, that provides rules, guidelines and/or features for common use, in order to obtain an optimal level of performance in a given context..." [9].

\section{On Economic Analysis}

Economic analysis supports health care policy and organizational decision making. However, it encounters some difficulties with eHealth systems, which are as follows: constantly changing technology, inconsistent study design to manage inadequate sample sizes, the inappropriateness of 
conventional techniques of economic evaluation, and the problem of placing value on health and nonhealth outcomes [10]. Consequently, five methods have been used to calculate the cost-effectiveness of traditional and eHealth interventions: cost-minimization analysis, cost-benefit analysis, cost-effectiveness analysis, cost-utility analysis, and cost-consequence analysis [11].

\section{Data Sources and Search Strategy}

This review of systematic reviews has been conducted in accordance with the preferred reporting items for systematic reviews and meta-analyses (PRISMA) statement [12] and the recommended methodological considerations when using existing systematic review as described by Whitlock et al [13].

On February 22, 2016, electronic searches were conducted on the MEDLINE, Cochrane Library, LILACS, and IEEE Library databases. To identify the EHR concept, standards for interoperability, and health information and its cost benefits, the search strategy was:

(("Electronic health records"[MeSH Terms] OR "Health Information Exchange"[MeSH Terms] OR ("Health Information Management"[MeSH Terms] OR ("Medical Informatics" [MeSH Terms]) AND ("Interoperability" OR "Standard of Information")) AND ("Cost-Benefit Analysis"[MeSh] OR "Evaluation Studies"[Publication Type] OR "Program Evaluation"[MeSh] OR impact or effectiveness)

The search was limited by language of publication (English, Spanish, French, Italian, and Portuguese), studies in humans, type of study (systematic reviews and meta-analyses), and year of publication (since 2005). Two systematic reviews that satisfied the criteria were identified manually. To better define certain eHealth technology descriptions, additional sources of evidence were considered.

\section{Study Selection}

The inclusion criteria were as follows:

1. Primary impact: EMR, standards, or interoperability on cost-benefit, or

2. Secondary impact: EMR, standards, or interoperability on quality of care (clinical outcomes), and

3. Real-life reviews about interventions in in-hospital settings.

Studies in primary or secondary care scenarios, studies without the primary or secondary impact of eHealth actions, and duplications were excluded. Titles and abstracts of retrieved papers were independently screened and evaluated by 2 investigators (ZSNR and TAM). Abstracts providing insufficient information were retrieved for independent, full-text evaluation by 2 investigators to determine study eligibility. Disagreements were resolved by consensus. Additional publications were identified using the reference lists of selected manuscripts.

\section{Data Extraction and Quality Assessment}

ZSNR prepared electronic data with paper contents abstracted using StArt software (Systematic Review System) to organize the analysis [14]. The data extraction of full-text analysis included the following: study design, number of studies evaluated, objectives, type of interventions/clinical data sources, eHealth interventions and terminology, interface/health information exchanges, duration of follow-up, cost-effectiveness, impact on quality of care, main results control group, potential bias, limitations, and lessons learned. The results were summarized into two subgroups according to the modality of intervention:

- Subgroup 1: eHealth systems implementation without health information exchange

- Subgroup 2: eHealth systems with health information exchange functionalities

The methodological quality assessment was based on the AMSTAR (a measurement tool to assess systematic reviews) checklist [15].

\section{Results}

A total of 288 papers were identified during the initial research phase, which decreased to 273 after removing 15 duplicates. After applying our criteria, only six systematic reviews were included in the final analysis and data-abstraction phase. The review process is represented in Figure 1, according PRISMA Statement [14].

The primary cause for excluding the 20 studies was mixed or outpatient settings for eHealth interventions (11 papers of 14 excluded, 79\%). The Pan American Health Organization (PAHO) conducted a review of the implementation and effective use of standards to achieve interoperability in Latin American and Caribbean countries but without direct or indirect outcomes analysis [6]. Multimedia Appendix 2 presents a detailed summary of the 14 full-text excluded systematic reviews.

\section{Characteristics and Quality of the Selected Studies}

Evidence of the cost-effectiveness of eHealth interventions that met the criteria was identified. Only one systematic review of the six performed a meta-analysis [16]. The quality assessment of the included studies followed AMSTAR (a measurement tool to assess systematic reviews) methodology and resulted in wide variability of the quality score. Two studies were classified with a moderate rating of quality with 5 positive points among 11 items [16,17], whereas other reports neglected many AMSTAR criteria [18-21]. Table 1 summarizes the quality assessment ratings, the study design, and the funding or support of the six included systematic reviews. 
Figure 1. Flow of information through the different phases of the systematic review.
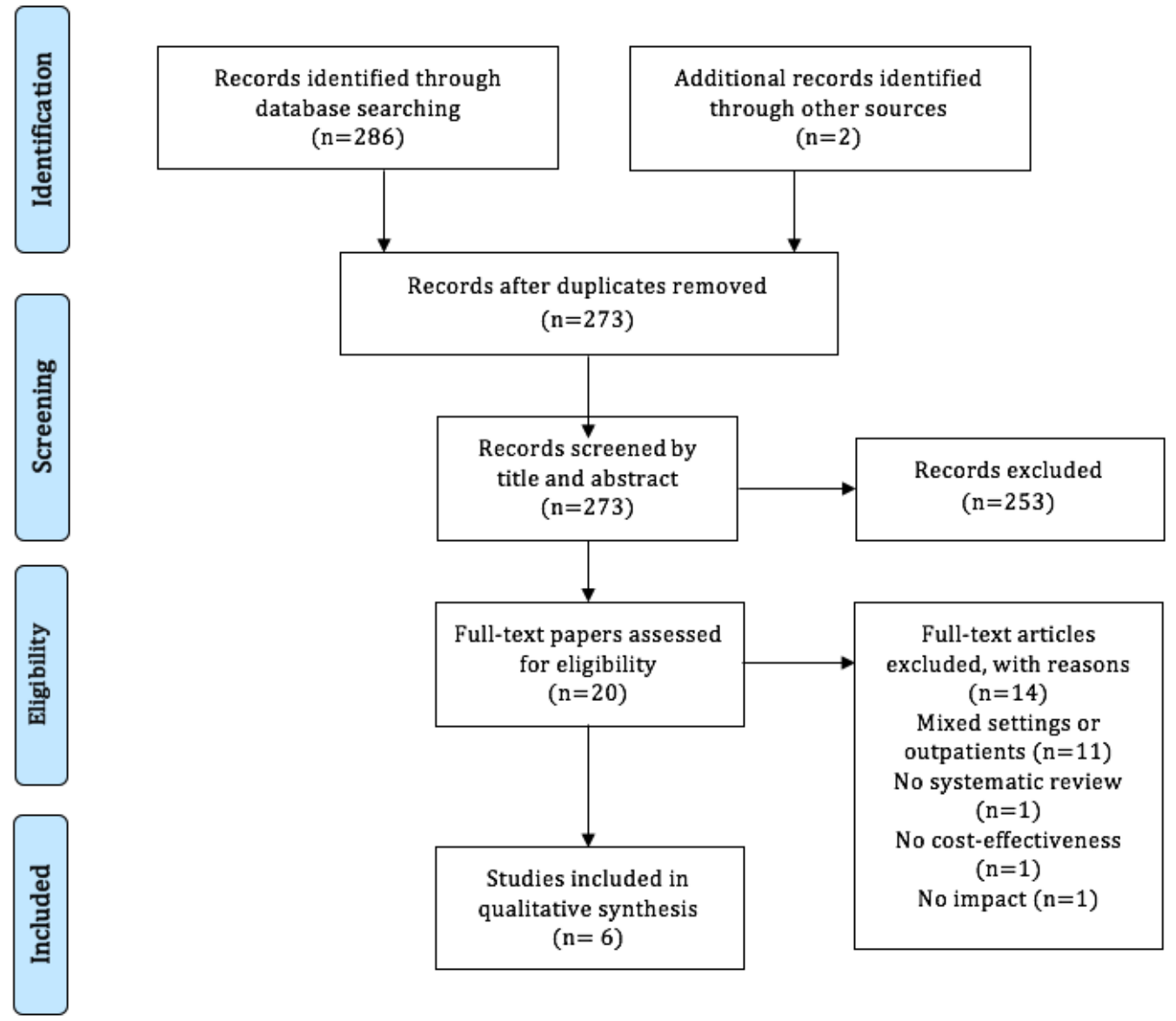
Table 1. Quality assessment ratings and characteristics of the six included systematic reviews.

\begin{tabular}{|c|c|c|c|c|c|c|c|c|c|}
\hline \multirow[t]{2}{*}{ Study } & \multicolumn{4}{|c|}{ AMSTAR $^{\mathrm{a}}$ score } & \multirow{2}{*}{$\begin{array}{l}\text { Funding or } \\
\text { support }\end{array}$} & \multirow[t]{2}{*}{ Study design } & \multirow{2}{*}{$\begin{array}{l}\text { Number of } \\
\text { studies evaluated }\end{array}$} & \multirow{2}{*}{$\begin{array}{l}\text { Control group } \\
\text { (most frequent) }\end{array}$} & \multirow{2}{*}{$\begin{array}{l}\text { Meta- } \\
\text { analysis }\end{array}$} \\
\hline & $\mathrm{Y}^{\mathrm{b}}$ & $\mathrm{N}^{\mathrm{c}}$ & $\mathrm{CA}^{\mathrm{d}}$ & $\mathrm{N} / \mathrm{A}^{\mathrm{e}}$ & & & & & \\
\hline Thompson et al 2015 [16] & 4 & 7 & 0 & 0 & $\mathrm{Y}$ & $\begin{array}{l}\mathrm{RCT}^{\mathrm{f}} \text {, pre-post studies, de- } \\
\text { scriptive studies }\end{array}$ & $\begin{array}{l}45 \text { total/Meta- } \\
\text { analysis: } 26\end{array}$ & $\begin{array}{l}\text { Pre-post imple- } \\
\text { mentation (pa- } \\
\text { per vs system) }\end{array}$ & $\mathrm{Y}$ \\
\hline Cheung et al 2015 [17] & 5 & 4 & 0 & 2 & $\mathrm{NC}^{\mathrm{g}}$ & $\begin{array}{l}\text { RCT, quasi-experimental } \\
\text { studies, descriptive studies }\end{array}$ & 18 & $\begin{array}{l}\text { Pre-post imple- } \\
\text { mentation }\end{array}$ & $\mathrm{N}$ \\
\hline de Bruin et al 2014 [20] & 2 & 7 & 0 & 2 & $\mathrm{NC}$ & Quasi-experimental & 26 & $\begin{array}{l}\text { True infection } \\
\text { detection by in- } \\
\text { fection control } \\
\text { experts }\end{array}$ & $\mathrm{N}$ \\
\hline Mapp et al 2013 [21] & 1 & 7 & 1 & 2 & $\mathrm{NC}$ & Observational, Pilot studies & 9 & No control & $\mathrm{N}$ \\
\hline Li et al 2013 [18] & 4 & 5 & 0 & 2 & $\mathrm{NC}$ & $\begin{array}{l}\text { RCT, quasi-experimental } \\
\text { studies }\end{array}$ & 6 & $\begin{array}{l}\text { Patient not re- } \\
\text { ported in writ- } \\
\text { ten notes or be- } \\
\text { fore system }\end{array}$ & $\mathrm{N}$ \\
\hline Govindan et al 2010 [19] & 5 & 4 & 0 & 2 & Y & $\begin{array}{l}\text { Observational: accuracy of } \\
\text { the automated method with } \\
\text { a gold standard method }\end{array}$ & 43 & $\begin{array}{l}\text { Standard chart } \\
\text { review }\end{array}$ & $\mathrm{N}$ \\
\hline
\end{tabular}

\footnotetext{
aMSTAR: a measurement tool to assess systematic reviews.

${ }^{\mathrm{b}} \mathrm{Y}$ : yes.

${ }^{\mathrm{c}} \mathrm{N}$ : no.

${ }^{\mathrm{d}} \mathrm{CA}$ : cannot answer.

${ }^{\mathrm{e}} \mathrm{N} / \mathrm{A}$ : not applicable.

${ }^{\mathrm{f}} \mathrm{RCT}$ : randomized controlled trial.

$\mathrm{g}_{\mathrm{NC}}$ : not commissioned.
}

Table 2 summarizes the objective, the type of intervention/clinical data sources, eHealth intervention and terminology, interface/health information exchange, and duration of follow-up of the six included systematic reviews.

\section{Summarized Outcomes}

Among the included systematic reviews, only one was classified as showing an effect on eHealth implementation without electronic health information exchange (Subgroup 1), and the other five were ranked as showing effects of systems implementation with incorporated health information exchange among other electronic data sources (Subgroup 2).

\section{Subgroup 1}

Considering eHealth systems implementation without health information exchange, the review of Thompson et al [16] reported a parallel to advances in digital technology and how different forms of eHealth systems have been developed and implemented (Table 3).

\section{Types and Functions of Technology Systems}

The selected review stated a mix of electronic interventions: EHR, EMR, computerized decision support systems (CDSS), computerized provider order-entry (CPOE) and surveillance systems used by physicians, nurses, allied health professionals, and managers of health services evaluating evidence from pre-and postsystems implementation. The analysis synthesized 46 publications about systems for diagnosis, treatment, and clinical monitoring. The study included a meta-analysis extracted from 26 publications to evaluate the effects of different types of systems regarding health IT in the inpatient of intensive care unit (ICU) setting on mortality, length of stay (LOS), and cost.

\section{Effects on Quality or Efficiency of Care}

Not enough evidence showed that electronic interventions can improve quality and safety of health care. The goals for secondary outcomes were the effects of health IT in the inpatient and ICU on mortality or LOS. The quality of included studies and interventions varied significantly, which was highlighted as the major limitation. Despite this, the surveillance systems had a pooled odd ratio (OR) of 0.85 (95\% CI 0.76-0.94) with moderate heterogeneity, $\mathrm{I}^{2}$ of $59 \%$.

\section{Effects on Costs}

Costs were unable to be evaluated quantitatively because the primary studies presented mixed and inconclusive results, leaving us unable to draw a definitive conclusion about cost-effectiveness. The analysis of costs was more limited than the evidence on quality and efficiency.

\section{Subgroup 2}

EHR implementation with health information exchange is a recent worldwide trend in hospital settings. A summary of the results of the systematic reviews included in subgroup 2 is presented in Table 4. 
Table 2. Descriptive summary of the systematic reviews included in electronic medical records (EMRs)/Interoperability review.

\begin{tabular}{|c|c|c|c|c|c|}
\hline Study & Objective & $\begin{array}{l}\text { Type of intervention/ } \\
\text { Clinical data sources }\end{array}$ & $\begin{array}{l}\text { eHealth intervention } \\
\text { and terminology }\end{array}$ & $\begin{array}{l}\text { Interface/health } \\
\text { information exchange }\end{array}$ & Duration of follow-up \\
\hline Thompson et al 2015 [16] & $\begin{array}{l}\text { To evaluate effects of } \\
\text { health } \mathrm{IT}^{\mathrm{a}} \text { in the inpa- } \\
\text { tient and } \mathrm{ICU}^{\mathrm{b}} \text { on } \\
\text { mortality, } \mathrm{LOS}^{\mathrm{c}}, \text { and } \\
\text { cost }\end{array}$ & $\begin{array}{l}\text { Multiple health IT inter- } \\
\text { ventions on diagnosis, } \\
\text { treatment, monitoring, } \\
\text { cost reduction/No refer- } \\
\text { ence }\end{array}$ & $\begin{array}{l}\mathrm{EHR}^{\mathrm{d}}, \mathrm{EMR}^{\mathrm{e}}, \\
\mathrm{CDSS}^{\mathrm{f}}, \mathrm{CPOE}^{\mathrm{g}}, \\
\text { Surveillance system }\end{array}$ & No reference & No reference \\
\hline Cheung et al 2015 [17] & $\begin{array}{l}\text { To evaluate the effects } \\
\text { of an information sys- } \\
\text { tem integrated to } \\
\text { PDMS } \\
\text { tional on organiza- } \\
\text { outcomes, in } \\
\text { ICUical }\end{array}$ & $\begin{array}{l}\text { Integrating bedside } \\
\text { equipment to an infor- } \\
\text { mation system/vital } \\
\text { signs, patient monitor, } \\
\text { ventilator, anesthesia } \\
\text { machine, dialysis ma- } \\
\text { chine, IV pump, lab } \\
\text { values, hospital informa- } \\
\text { tion system, admission, } \\
\text { discharge and transfer }\end{array}$ & $\begin{array}{l}\text { CDSS, PDMS, health } \\
\text { information exchange }\end{array}$ & $\begin{array}{l}\text { PDMS to an informa- } \\
\text { tion system/no men- } \\
\text { tion about direction of } \\
\text { data exchange }\end{array}$ & $\begin{array}{l}1 \text { day to } 1 \text { week; } 11 \\
\text { months to } 4 \text { years }\end{array}$ \\
\hline de Bruin et al 2014 [20] & $\begin{array}{l}\text { To evaluate recent } \\
\text { trends in use of elec- } \\
\text { tronically available } \\
\text { patient data by elec- } \\
\text { tronic surveillance } \\
\text { systems for HAIs }{ }^{\mathrm{j}} \text { and } \\
\text { identify consequences } \\
\text { for system effective- } \\
\text { ness }\end{array}$ & $\begin{array}{l}\text { HAIs that utilize EHR } \\
\text { available in hospitals to } \\
\text { surveillance the } \\
\text { HAIs/Medico-adminis- } \\
\text { trative data procedures } \\
\text { or discharge reports, } \\
\text { free text reports, bio- } \\
\text { chemistry, microbiolo- } \\
\text { gy, and radiology labo- } \\
\text { ratory test results, phar- } \\
\text { macy dispensing } \\
\text { records, radiology free- } \\
\text { text records, vital signs, } \\
\text { electronic discharge } \\
\text { summary }\end{array}$ & $\begin{array}{l}\text { Automated detection } \\
\text { by HAI systems: } \\
\text { EHR, health informa- } \\
\text { tion exchange, using } \\
\text { ICD }^{\mathrm{k}}-9 \text {, ICD-10, dis- } \\
\text { charge coding, ATC } \\
\text { code }\end{array}$ & $\begin{array}{l}\text { EHR to HAI sys- } \\
\text { tems/no mention } \\
\text { about direction of data } \\
\text { exchange }\end{array}$ & No reference \\
\hline Mapp et al 2013 [21] & $\begin{array}{l}\text { To examine early } \\
\text { warning scoring sys- } \\
\text { tems and their effec- } \\
\text { tiveness in predicting } \\
\text { a patient's potential } \\
\text { for deterioration and } \\
\text { considers whether } \\
\text { these scoring systems } \\
\text { prevent unplanned } \\
\text { ICU admissions } \\
\text { and/or death }\end{array}$ & $\begin{array}{l}\text { Instruments and clinical } \\
\text { support systems avail- } \\
\text { able to assist health care } \\
\text { personnel in recogniz- } \\
\text { ing early clinical deteri- } \\
\text { oration/Vital signs, } \\
\mathrm{SpO}_{2}{ }^{\mathrm{m}}, \mathrm{LOC}^{\mathrm{n}}, \mathrm{UOP}^{\mathrm{o}} \text {, } \\
\text { nurse/family concerns, } \\
\text { complaints, lab values }\end{array}$ & $\begin{array}{l}\text { EMR, CDSS, health } \\
\text { information exchange } \\
\text { based on } \mathrm{SBAR}^{\mathrm{p}} \\
\text { communication }\end{array}$ & $\begin{array}{l}\text { Early warning scoring } \\
\text { systems that interface } \\
\text { with EMRs and are } \\
\text { supplemented with } \\
\text { decision aides (algo- } \\
\text { rithms) and clinical } \\
\text { support systems/no } \\
\text { mention about direc- } \\
\text { tion of data exchange }\end{array}$ & $\begin{array}{l}\text { Seven studies: } 3 \text { to } 15 \\
\text { months/two studies: } \\
\text { over } 24 \text { months to } 8 \\
\text { years }\end{array}$ \\
\hline Li et al 2013 [18] & $\begin{array}{l}\text { To evaluate the im- } \\
\text { pact of the CHTs }{ }^{\mathrm{q}} \text { on } \\
\text { the quality of physi- } \\
\text { cian handoff, patient } \\
\text { care, and physician } \\
\text { work efficiency }\end{array}$ & $\begin{array}{l}\text { Decision support/train- } \\
\text { ing, emergency refer- } \\
\text { rals, supervision, alerts } \\
\text { and reminders, client } \\
\text { education, data collec- } \\
\text { tion, medicine dos- } \\
\text { ing/Patient demograph- } \\
\text { ics, medications, diagno- } \\
\text { sis, problem lists, com- } \\
\text { ment line, vital signs, } \\
\text { to-do list, LOS, free } \\
\text { daily notes, lab values }\end{array}$ & $\begin{array}{l}\text { CHTs, EMR, CDSS, } \\
\text { health information ex- } \\
\text { change. Allergy Code }\end{array}$ & $\begin{array}{l}\text { Clinical information } \\
\text { exchange using CHTs } \\
\text { for physician handoff } \\
\text { for hospitalized pa- } \\
\text { tients CHTs/mixed } \\
\text { (no interface, unidirec- } \\
\text { tional or bidirectional } \\
\text { interface exchange) }\end{array}$ & 1 to 6 months \\
\hline Govindan et al 2010 [19] & $\begin{array}{l}\text { To identify, describe, } \\
\text { and evaluate the effec- } \\
\text { tiveness of automated } \\
\text { inpatient harm-detec- } \\
\text { tion methods }\end{array}$ & $\begin{array}{l}\text { Automated harm detec- } \\
\text { tion on EMR. Gold } \\
\text { standard: chart review }\end{array}$ & $\begin{array}{l}\text { Automated detection } \\
\text { by surveillance sys- } \\
\text { tems: EMR, health in- } \\
\text { formation exchange, } \\
\text { using ICD-9, proce- } \\
\text { dure codes, billing } \\
\text { codes }\end{array}$ & $\begin{array}{l}\text { Automated harm de- } \\
\text { tection on EMR, using } \\
\text { field-defined systems, } \\
\text { natural language-pro- } \\
\text { cessing/Unidirectional } \\
\text { retrospective }\end{array}$ & No reference \\
\hline
\end{tabular}


aT: information technology.

bICU: intensive care unit.

${ }^{c}$ LOS: length of stay.

${ }^{\mathrm{d}}$ EHR: electronic health record.

${ }^{\mathrm{e}}$ EMR: electronic medical record.

${ }^{\mathrm{f}} \mathrm{CDSS}$ : computerized decision support systems.

${ }^{\mathrm{g}}$ CPOE: computerized provider order-entry.

${ }^{h}$ PDMS: Patient data management system.

${ }^{\mathrm{i}}$ ICU: intensive care unit.

${ }^{\mathrm{j}}$ HAIs: health care-associated infections systems.

${ }^{\mathrm{k}} \mathrm{ICD}$ : international classification of disease.

${ }^{\mathrm{i}}$ ATC: anatomical therapeutic chemical.

${ }^{\mathrm{m}} \mathrm{SpO}_{2}$ : oxygen saturation.

${ }^{\mathrm{n}}$ LOC: level of consciousness.

${ }^{\circ}$ UOP: urine output.

${ }^{\mathrm{p}} \mathrm{SBAR}$ : situation, background, assessment, recommendation.

${ }^{\mathrm{q}}$ CHTs: computerized physician handoff tools.

Table 3. Descriptive summary of the results of systematic reviews included in electronic medical records(EMRs)/Interoperability review. Subgroup 1: electronic health (eHealth) systems implementation without health information exchange.

\begin{tabular}{|c|c|c|c|c|c|}
\hline Study & $\begin{array}{l}\text { Primary impact: } \\
\text { Cost-effectiveness }\end{array}$ & $\begin{array}{l}\text { Secondary impact: } \\
\text { Quality of care/ } \\
\text { Clinical outcome }\end{array}$ & Main results & Potential bias & Lessons \\
\hline Thompson et al 2015 [16] & $\begin{array}{l}\text { Mixed and inconclu- } \\
\text { sive }\end{array}$ & $\begin{array}{l}\text { Mortality: overall } \\
\text { CPOE }^{\mathrm{a}} \text { systems did not } \\
\text { show a significant ef- } \\
\text { fect }\left(\mathrm{OR}^{\mathrm{b}}: 0.91,95 \%\right. \\
\left.\text { CI } 0.75-1.10 ; \mathrm{I}^{2 \mathrm{c}} 66 \%\right) \text {, } \\
\text { nor EHR }{ }^{\mathrm{d}} \text { alone (OR: } \\
0.96,95 \% \text { CI 0.77- } \\
\text { 1.19). CDSS }(\mathrm{OR} 0.96 \text {, } \\
\text { 95\% CI 0.77-1.19). The } \\
\text { surveillance systems } \\
\text { had a pooled OR of } \\
0.85 \text { (95\% CI 0.76- } \\
0.94) \text { with moderate } \\
\text { heterogeneity, I²59\% } \\
\text { LOS: CPOE trended to- } \\
\text { ward a reduction in } \\
\text { LOS (mean decrease, } \\
0.67 \text { days, } 95 \% \text { CI } \\
\text {-2.07 to } 0.73) \text {, though } \\
\text { with significant hetero- } \\
\left.\text { geneity (I }{ }^{2} 82 \%\right) \text {. Nei- } \\
\text { ther CDSS nor surveil- } \\
\text { lance systems trended } \\
\text { toward changes in hos- } \\
\text { pital LOS, and the net- } \\
\text { pooled effect was not } \\
\text { significant. }\end{array}$ & $\begin{array}{l}\text { Electronic interven- } \\
\text { tions were not shown } \\
\text { to have a substantial } \\
\text { effect on mortality, } \\
\text { LOS }^{f} \text {, or cost. }\end{array}$ & $\begin{array}{l}\text { Selection, measure- } \\
\text { ment }\end{array}$ & $\begin{array}{l}\text { There is not enough } \\
\text { evidence to confident- } \\
\text { ly state that electronic } \\
\text { interventions have the } \\
\text { ability to achieve the } \\
\text { goal of improving } \\
\text { quality and safety. }\end{array}$ \\
\hline
\end{tabular}

${ }^{\mathrm{a}} \mathrm{CPOE}$ : computerized provider order-entry.

${ }^{\mathrm{b}} \mathrm{OR}$ : odds ratio.

${ }^{\mathrm{c}} \mathrm{I}^{2}$ : measure of heterogeneity.

${ }^{\mathrm{d}}$ EHR: electronic health record.

${ }^{\mathrm{e}} \mathrm{CDSS}$ : computerized decision support systems.

${ }^{\mathrm{f}} \mathrm{LOS}$ : length of stay. 
Table 4. Descriptive summary of the results of systematic reviews included in the electronic health record (EHR)/Interoperability review. Subgroup 2: electronic health (eHealth) systems implementation with information exchange.

\begin{tabular}{|c|c|c|c|c|c|}
\hline Study & $\begin{array}{l}\text { Primary impact: } \\
\text { Cost-effectiveness }\end{array}$ & $\begin{array}{l}\text { Secondary impact: } \\
\text { Quality of care/ } \\
\text { Clinical outcome }\end{array}$ & Main results & Potential bias & Lessons \\
\hline Cheung et al 2015 [17] & Not evaluated ${ }^{\mathrm{a}}$ & $\begin{array}{l}\text { PDMS }{ }^{b} \text { reduced chart- } \\
\text { ing time, increased time } \\
\text { spent on direct patient } \\
\text { care and reduced the } \\
\text { occurrence of errors } \\
\text { (medication errors, intra- } \\
\text { venous and ventilation } \\
\text { incidents). The effect } \\
\text { on documentation was } \\
\text { mixed. Improvement in } \\
\text { clinical outcomes when } \\
\text { PDMS was integrated } \\
\text { with a CDSS } \text {, but } \\
\text { scarce literature is } \\
\text { available. }\end{array}$ & $\begin{array}{l}\text { The effect on docu- } \\
\text { mentation was mixed. } \\
\text { Qualitative analysis } \\
\text { showed a significant } \\
\text { decrease in time spent } \\
\text { on documentation. } \\
\text { Clinical outcomes: in- } \\
\text { conclusive. }\end{array}$ & $\begin{array}{l}\text { Selection, measure- } \\
\text { ment }\end{array}$ & $\begin{array}{l}\text { Improvement in clini- } \\
\text { cal outcomes when } \\
\text { PDMS was integrated } \\
\text { with a CDSS, but } \\
\text { there is scarce litera- } \\
\text { ture available. Organi- } \\
\text { zational advantages } \\
\text { included improved ac- } \\
\text { curacy, legibility, data } \\
\text { accessibility, and deci- } \\
\text { sion support. Such in- } \\
\text { tegration may im- } \\
\text { prove clinical out- } \\
\text { comes, although fur- } \\
\text { ther studies are re- } \\
\text { quired for validation. }\end{array}$ \\
\hline de Bruin et al 2014 [20] & Not evaluated ${ }^{\mathrm{a}}$ & $\begin{array}{l}\text { Electronic surveillance } \\
\text { achieves equal or better } \\
\text { sensitivity than manual } \\
\text { surveillance. Several } \\
\text { studies also reported } \\
\text { time savings of } 60 \% \text { to } \\
99.9 \% \text { or a reduction in } \\
\text { chart reviews of } 40 \% \text { to } \\
90.5 \% \text {. }\end{array}$ & $\begin{array}{l}\text { Driven by the in- } \\
\text { creased availability of } \\
\text { electronic patient data, } \\
\text { electronic } \\
\text { HAIs }{ }^{d} \text { surveillance } \\
\text { systems use more da- } \\
\text { ta, making systems } \\
\text { more sensitive yet less } \\
\text { specific but also allow } \\
\text { systems to be tailored } \\
\text { to the needs of health } \\
\text { care institutes' } \\
\text { surveillance pro- } \\
\text { grams. }\end{array}$ & Selection & $\begin{array}{l}\text { HAIs detection sys- } \\
\text { tems use increasingly } \\
\text { more EHR }{ }^{\mathrm{e}} \text { and patient } \\
\text { data as more data } \\
\text { sources become avail- } \\
\text { able. Thus, systems } \\
\text { tend to become more } \\
\text { sensitive and less spe- } \\
\text { cific. }\end{array}$ \\
\hline
\end{tabular}

\begin{tabular}{|c|c|c|c|c|c|}
\hline Mapp et al 2013 [21] & Not evaluated $^{\mathrm{a}}$ & $\begin{array}{l}\text { An increase occurred in } \\
\text { the number of rapid re- } \\
\text { sponse calls by nursing } \\
\text { staff, a decrease in un- } \\
\text { planned ICU admis- } \\
\text { sions, and a decrease in } \\
\text { hospital mortality. }\end{array}$ & $\begin{array}{l}\text { Improvement in clini- } \\
\text { cal outcomes when } \\
\text { using early warning } \\
\text { scoring systems. }\end{array}$ & Selection & $\begin{array}{l}\text { Early warning scoring } \\
\text { systems can be more } \\
\text { effective with the inte- } \\
\text { gration of algorithms } \\
\text { and clinical support } \\
\text { systems. }\end{array}$ \\
\hline Li et al 2013 [18] & Not evaluated ${ }^{\mathrm{a}}$ & $\begin{array}{l}\text { Impact on physician } \\
\text { work efficiency (self- } \\
\text { reported time spent on } \\
\text { handing copying patient } \\
\text { information; } 50 \% \text { ) and } \\
\text { proportionally more } \\
\text { time to see patients. } \\
\text { Time on each patient } \\
\text { during rounding de- } \\
\text { creased by } 1.5 \text { min. Im- } \\
\text { pact on quality on } \\
\text { physician handoff: } \\
\text { completeness and con- } \\
\text { sistency of the handoff } \\
\text { document has im- } \\
\text { proved. }\end{array}$ & $\begin{array}{l}\text { Completeness and } \\
\text { consistency of the } \\
\text { handoff document has } \\
\text { improved. Accuracy } \\
\text { of information about } \\
\text { patients during physi- } \\
\text { cian handoff. }\end{array}$ & $\begin{array}{l}\text { Selection, measure- } \\
\text { ment }\end{array}$ & $\begin{array}{l}\text { CHTs }^{g} \text { could potential- } \\
\text { ly enhance work effi- } \\
\text { ciency and continuity } \\
\text { of care during physi- } \\
\text { cian handoff, but the } \\
\text { role in improving } \\
\text { quality is less clear. } \\
\text { The information } \\
\text { available was often } \\
\text { not sufficient to help } \\
\text { on-call physicians } \\
\text { make patient care deci- } \\
\text { sions. }\end{array}$ \\
\hline
\end{tabular}




\begin{tabular}{|c|c|c|c|c|c|}
\hline Study & $\begin{array}{l}\text { Primary impact: } \\
\text { Cost-effectiveness }\end{array}$ & $\begin{array}{l}\text { Secondary impact: } \\
\text { Quality of care/ } \\
\text { Clinical outcome }\end{array}$ & Main results & Potential bias & Lessons \\
\hline Govindan et al 2010 [19] & Not evaluated ${ }^{\mathrm{a}}$ & 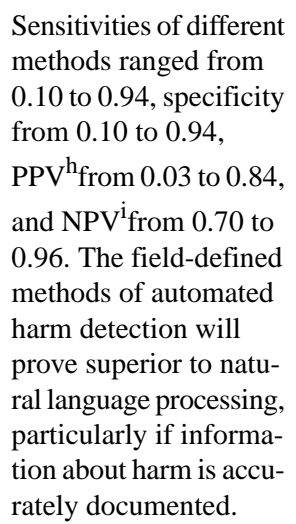 & $\begin{array}{l}\text { Automated harm de- } \\
\text { tection has the poten- } \\
\text { tial to positively influ- } \\
\text { ence clinical practice. }\end{array}$ & $\begin{array}{l}\text { Selection, measure- } \\
\text { ment }\end{array}$ & $\begin{array}{l}\text { Automated harm de- } \\
\text { tection has the poten- } \\
\text { tial to positively influ- } \\
\text { ence clinical practice. } \\
\text { Another potential } \\
\text { benefit is the reduc- } \\
\text { tion of person-hour } \\
\text { required to harm } \\
\text { surveillance. }\end{array}$ \\
\hline
\end{tabular}

\footnotetext{
${ }^{a}$ Not evaluated in the selected study.

bPDMS: Patient data management system.

${ }^{\mathrm{c}}$ CDSS: computerized decision support systems.

${ }^{\mathrm{d}}$ HAIs: health care-associated infections systems.

${ }^{\mathrm{e}}$ EHR: electronic health record.

${ }^{f}$ ICU: intensive care unit.

${ }^{g}$ CHTs: computerized physician handoff tools

${ }^{\mathrm{h}} \mathrm{PPV}$ : positive predictive value.

${ }^{\mathrm{i}} \mathrm{NPV}$ : negative predictive value.
}

\section{Types and Functions of Technology Systems}

Most of the reviews use ICUs as settings for eHealth intervention analysis. However, the objectives of interventions were quite heterogeneous. Two studies reported the effect of surveillance systems on harm detection [19] and health care-associated infections [20]. Bedside data integration in an information system [17], continuity of care using physician handoff tools [18], and prediction of death or unexpected ICU admission [21] were the proposals of the other reviews. Regarding application users, two studies focused on patient outcome results for health care managers $[19,20]$. Some focused directly on health care professionals to improve clinical practice $[18,19,21]$. On the direction of electronic health information exchange, one review described it as unidirectional [19], three did not clarify whether the exchange was bidirectional [17,20,21], and one summarized mixed studies including systems without interfaces [18]. None mentioned interoperability among electronic health systems. Regarding standards for the exchange of clinical data, four studies reported the use of terminologies such as International Classification of Disease (ICD) and anatomical therapeutic chemical (ATC) code [18-21].

\section{Effects on Quality or Efficiency of Care}

Among reviews focused on improving clinical practice, inconclusive results in direct patient care were reported by Cheung et al [17]. Mapp et al [21] highlighted an increase in nursing staff efficiency regarding rapid calls response, a decrease in unplanned ICU admissions, and hospital mortality. Li et al [18] presented a positive impact on continuity of inpatient care. With regard to indirect results on patient care, two studies highlighted the improvement of health data quality in terms of

accuracy, legibility, completeness, and consistency of documents $[17,18]$. The other reviews focused on electronic surveillance. The results showed that systems tend to become more sensitive and less specific than manual monitoring to detect infection [20]. With respect to inpatient harm detection, the automated systems allowed rapid scanning of a vast number of patient records with minimal effort and may identify events as they occur in real time [19]. Most automated surveillance systems were retrospective, but some real-time surveillance alerts that informed physicians and pharmacists of adverse events were reported [19].

\section{Effects on Costs}

None of the reviews evaluated effects of eHealth interventions on costs.

\section{Discussion}

\section{Principal Findings}

This study found preliminary benefits in the use of electronically available inpatient data systems on the quality of care. Despite the limited number of studies that met the eligibility criteria, the heterogeneity of electronic systems reported, and different interventions on hospital routines, the identification of preliminary secondary benefits on patient mortality was possible [16]. eHealth systems with information exchange functionalities also showed potential impact on quality of care or patient outcomes. From five studies, one had inconclusive results on direct patient care [17] and four presented partial effects, as nursing staff efficiency led to a faster call response, a decrease in unplanned ICU admissions and hospital mortality [18], improvement of health data quality $[17,18]$, and more efficient 
surveillance programs inside hospitals $[19,20]$. It is expected that the systems able to share health information would improve care at the time and point of attention, especially the surveillance systems and those that use common terminologies and vocabularies to support consistency in information collection $[6,19,20,22]$.

However, no substantial review regarding the impact of electronic interventions on cost-effectiveness was identified. Among the six analyses included, only Thompson et al reported that some preliminary studies have identified decreases in cost, but the heterogeneity and the absence of information of follow-up impaired a proper analysis of cost-effectiveness [16]. Immediate cost savings are not anticipated for organizations when choosing to adopt eHealth strategies because the high cost of implementation limits the transition from paper-based to electronic systems and represents a significant challenge to their widespread adoption [23]. Regardless, medium and long-term positive results are expected, and the World Health Organization (WHO) recognized overall eHealth as cost- effective and secure [24]. Potential indirect cost saving was mentioned as a secondary outcome in three studies, with the reduction of person-hours harming surveillance and the increase in time spent on direct patient care [17,18,24].

Unfortunately, no study about interoperability, in the sense of syntactic and semantic meaning, on cost benefit was identified. Importantly, none of the studies in this review properly defined EHR concept as a longitudinal health record with entries by health care practitioners in multiple sites of care or mentioned interoperability applications among electronic systems. However, taking the antecedent step toward full interoperability, an effective information sharing between stakeholders and systems can be attained through the use of standards [6]. Standards adoption for the exchange of clinical data was mentioned in four studies [18-21], mostly terminologies adoptions, but the potential impact of such tools on continuity of care or costs remains an open question that needs investigation. Although within the limits of hospital systems, the analysis confirmed the potential to positively impact physician practice organizations, as previously reported [23].
Further longitudinal research is needed to determine the actual impact of eHealth adoption on health care costs and clinical outcomes.

\section{Limitations}

The current results should be interpreted as a whole with the study limitations. Only four major databases were searched and gray literature sources were not evaluated. Additionally, the limitation to English, Spanish, French, Italian, and Portuguese languages prevented the capture of all relevant studies. Furthermore, the quality of included studies was poor, and they varied regarding the type of eHealth interventions, follow-up time, and goals. This systematic review summarized primary and secondary outcomes from different classes of intervention from which to draw results, analysis, and conclusions. Due to the variation in scenarios and lack of numeric goals, a meta-analysis was considered inappropriate.

\section{Conclusions and Lessons Learned}

This review identified some benefits on the quality of care but did not provide evidence that the eHealth interventions had a measurable impact on cost-effectiveness, mortality, or LOS in hospital settings. Preliminary evidence indicates that the use of eHealth interventions with information exchange may improve clinical process outcomes. The absence of studies precludes the assessment of impact of interoperability on benefits of health care or cost, and this aspect needs further research. Technological barriers might influence eHealth solutions implementation and data exchange for systems integration or interoperable interfaces. There are also issues with the lack of standardization of most aspects of health information and misuse of terms in the scientific publications. Authors should be explicit when they are using interfacing syntactic interoperability or semantic interoperability to reduce the confusion with different health information exchange possibilities. Further research with long-term follow-up is needed to determine the actual impact of eHealth adoption on health care costs to demonstrate (1) value for money (including clinical impacts) and (2) the clinical impact of semantic and synthetic interoperability.

\section{Acknowledgments}

We thank the team who worked with us on a systematic review with a large scope: Maria Beatriz Moreira Alkmim, Renato Minelli Figueira, João Antonio de Queiroz Oliveira, Gustavo Moreira Alkmim, Maria Clara Noman de Alencar, Nayara Dornela Quintino, and the undergratuate students-Edson Alexandre Silva Carvalho, Fernanda Cotrim Stefanelli, Gabriel Almeida Silqueira Rocha, Grace Kelly Matos e Silva, Marcelo Henriques de Camargos, Thales Matheus Mendonça Santos, Thiago Adriano de Deus Queiroz Santos, and Elsy Dumit Bechara.

Francisco Becerra-Posada and David Novillo-Ortiz are staff members of the PAHO/WHO and are themselves alone responsible for the views expressed in the paper, which does not necessarily represent the views, decisions, or policies of the PAHO/WHO.

\section{Conflicts of Interest}

None declared.

\section{Multimedia Appendix 1}

eHealth concepts and definitions. 


\section{Multimedia Appendix 2}

Descriptive summary of the 14 full-text excluded systematic reviews.

[PDF File (Adobe PDF File), 41KB-Multimedia Appendix 2]

\section{References}

1. World Health Organization. Apps.WHO. 2006. Building foundations for eHealth : progress of member states : report of the Global Observatory for eHealth URL: http://apps.who.int/iris/handle/10665/43599 [accessed 2017-07-13] [WebCite Cache ID 6rw1isUOl]

2. World Health Organization. Apps.WHO. 2006. Electronic health records: manual for developing countries URL: http:/ lapps.who.int/iris/handle/10665/207504 [accessed 2017-07-13] [WebCite Cache ID 6sm2rrOne]

3. Shekelle PG, Morton SC, Keeler EB. Costs and benefits of health information technology. Evid Rep Technol Assess (Full Rep) 2006 Apr(132):1-71. [Medline: 17627328]

4. World Health Organization. WHO. Geneve: WHO Press; 2016. Atlas of eHealth country profiles: the use of eHealth in support of universal health coverage: based on the findings of the third global survey on eHealth URL: http://www.who.int/ goe/publications/atlas 2015/en/ [accessed 2017-07-14] [WebCite Cache ID 6rF0c5lHC]

5. ncbi.nlm. 2017. Health Information Exchange URL: https://www.ncbi.nlm.nih.gov/mesh/?term=health+information+exchange [accessed 2017-06-20] [WebCite Cache ID 6rMNCkzRG]

6. Pan American Health Organization. Webcitation.: Pan American Health Organization; 2016. eHealth in Latin Americathe Caribbean: interoperability standards review URL: http://www.webcitation.org/6rwrfeqBJ [accessed 2017-07-14] [WebCite Cache ID 6rwrfeqBJ]

7. Webcitation. 2005. Interoperability Definition and Background URL: http://www.webcitation.org/6rKvIpuvO [accessed 2017-07-14] [WebCite Cache ID 6rKvIpuvO]

8. International Organization for Standarization - ISO. ISO. Health informatics -- Requirements for an electronic health record architecture URL: https://www.iso.org/standard/52823.html [accessed 2017-06-19] [WebCite Cache ID 6rL0KEbKn]

9. International Organization for Standarization - ISO. Webcitation. Standardization and related activities -- General vocabulary URL: http://www.webcitation.org/6rKvaBnAJ [accessed 2017-07-14] [WebCite Cache ID 6rKvaBnAJ]

10. Mistry H. Systematic review of studies of the cost-effectiveness of telemedicine and telecare. Changes in the economic evidence over twenty years. J Telemed Telecare 2012 Jan;18(1):1-6. [doi: 10.1258/jtt.2011.110505] [Medline: 22101609]

11. Rojas SV, Gagnon M. A systematic review of the key indicators for assessing telehomecare cost-effectiveness. Telemed J E Health 2008 Nov;14(9):896-904 [FREE Full text] [doi: 10.1089/tmj.2008.0009] [Medline: 19035798]

12. Liberati A, Altman D, Tetzlaff J, Mulrow C, Gøtzsche P, Ioannidis J. The PRISMA statement for reporting systematic reviews and meta-analyses of studies that evaluate healthcare interventionsxplanation and elaboration. Br Med J 2009;339:b2700 [FREE Full text] [doi: 10.1371/journal.pmed.1000100]

13. Whitlock EP, Lin JS, Chou R, Shekelle P, Robinson KA. Using existing systematic reviews in complex systematic reviews. Ann Intern Med 2008 May 20;148(10):776-782. [Medline: 18490690]

14. Hernandes E, Zamboni A, Di Thommazo A, Fabbri S. Avaliação da ferramenta StArt utilizando o modelo TAM e o paradigma GQM. In: Proceedings of 7th Experimental Software Engineering Latin American Workshop (ESELAW 2010). Goiânia: UFG/INF/FUNAPE; 2010 Presented at: 7th Experimental Software Engineering Latin American Workshop (ESELAW 2010); 12/10/2010; Goiânia URL: http://www.portal.inf.ufg.br/ eselaw2010/proceedings-eselaw2010.pdf\#page=37

15. Shea BJ, Grimshaw JM, Wells GA, Boers M, Andersson N, Hamel C, et al. Development of AMSTAR: a measurement tool to assess the methodological quality of systematic reviews. BMC Med Res Methodol 2007 Feb 15;7:10 [FREE Full text] [doi: 10.1186/1471-2288-7-10] [Medline: 17302989]

16. Thompson G, O'Horo JC, Pickering BW, Herasevich V. Impact of the electronic medical record on mortality, length of stay, and cost in the hospital and ICU: a systematic review and meta-analysis. Crit Care Med 2015 Jun;43(6):1276-1282. [doi: 10.1097/CCM.0000000000000948] [Medline: 25756413]

17. Cheung A, van Velden FH, Lagerburg V, Minderman N. The organizational and clinical impact of integrating bedside equipment to an information system: a systematic literature review of patient data management systems (PDMS). Int J Med Inform 2015 Mar;84(3):155-165. [doi: 10.1016/j.ijmedinf.2014.12.002] [Medline: 25601332]

18. Li P, Ali S, Tang C, Ghali WA, Stelfox HT. Review of computerized physician handoff tools for improving the quality of patient care. J Hosp Med 2013 Aug;8(8):456-463. [doi: 10.1002/jhm.1988] [Medline: 23169534]

19. Govindan M, Van Citters AD, Nelson EC, Kelly-Cummings J, Suresh G. Automated detection of harm in healthcare with information technology: a systematic review. Qual Saf Health Care 2010 Oct;19(5):e11. [doi: 10.1136/qshc.2009.033027] [Medline: 20671081]

20. de Bruin JS, Seeling W, Schuh C. Data use and effectiveness in electronic surveillance of healthcare associated infections in the 21st century: a systematic review. J Am Med Inform Assoc 2014;21(5):942-951 [FREE Full text] [doi: 10.1136/amiajnl-2013-002089] [Medline: 24421290]

21. Mapp ID, Davis LL, Krowchuk H. Prevention of unplanned intensive care unit admissions and hospital mortality by early warning systems. Dimens Crit Care Nurs 2013;32(6):300-309. [doi: 10.1097/DCC.0000000000000004] [Medline: 24100432] 
22. Hammond WE, Bailey C, Boucher P, Spohr M, Whitaker P. Connecting information to improve health. Health Aff (Millwood) 2010 Feb;29(2):284-288 [FREE Full text] [doi: 10.1377/hlthaff.2009.0903] [Medline: 20348075]

23. Police RL, Foster T, Wong KS. Adoption and use of health information technology in physician practice organisations: systematic review. Inform Prim Care 2010;18(4):245-258 [FREE Full text] [Medline: 22040851]

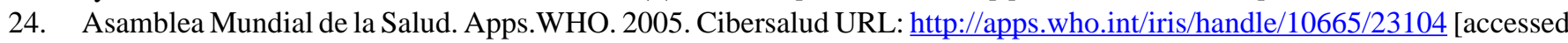
2017-07-14] [WebCite Cache ID 6smbcRPBE]

25. Kawamoto K, Houlihan C, Balas E, Lobach D. Improving clinical practice using clinical decision support systems: a systematic review of trials to identify features critical to success. Br Med J 2005;330(7494):765. [doi: 10.1136/bmj.38398.500764.8F]

26. Mitchell J. From Telehealth to E-health: The Unstoppable Rise of E-health. Canberra, NSW: Commonwealth Department of Communications, Information Technology and the Arts; 1999:064275036.

27. Eysenbach G. What is e-health? J Med Internet Res 2001 Jun;3(2):E20 [FREE Full text] [doi: 10.2196/jmir.3.2.e20] [Medline: 11720962$]$

28. Pan American Health Organization. PAHO. Washington, DC: PAHO HQ Library Cataloguing-in-Publication; 2011. Epidemiological Surveillance of Healthcare Associated Infections URL: http://new.paho.org/hq/dmdocuments/2011/ ENG_Modulo_I_final.pdf [accessed 2017-07-14] [WebCite Cache ID 6rwqNuepz]

29. Main C, Moxham T, Wyatt J, Kay J, Anderson R, Stein K. Computerised decision support systems in order communication for diagnostic, screening or monitoring test ordering: systematic reviews of the effects and cost-effectiveness of systems. Health Technol Assess 2017 Jul 14;14(48):1-227 [FREE Full text] [doi: 10.3310/hta14480] [Medline: 21034668]

30. Chaudhry B, Wang J, Wu S, Maglione M, Mojica W, Roth E, et al. Systematic review: impact of health information technology on quality, efficiency, and costs of medical care. Ann Intern Med 2006 May 16;144(10):742-752. [Medline: 16702590]

31. Jamal A, McKenzie K, Clark M. The impact of health information technology on the quality of medical and health care: a systematic review. HIM J 2009;38(3):26-37. [Medline: 19875852]

32. Wollersheim D, Sari A, Rahayu W. Archetype-based electronic health records: a literature review and evaluation of their applicability to health data interoperability and access. HIM J 2009;38(2):7-17. [Medline: 19546483]

33. Chan AJ, Chan J, Cafazzo JA, Rossos PG, Tripp T, Shojania K, et al. Order sets in health care: a systematic review of their effects. Int J Technol Assess Health Care 2012 Jul;28(3):235-240. [doi: 10.1017/S0266462312000281] [Medline: 22980699]

34. Sheehan J, Sherman KA. Computerised decision aids: a systematic review of their effectiveness in facilitating high-quality decision-making in various health-related contexts. Patient Educ Couns 2012 Jul;88(1):69-86. [doi: 10.1016/j.pec.2011.11.006] [Medline: 22185961]

35. Ojeleye O, Avery A, Gupta V, Boyd M. The evidence for the effectiveness of safety alerts in electronic patient medication record systems at the point of pharmacy order entry: a systematic review. BMC Med Inform Decis Mak 2013 Jul 01;13:69 [FREE Full text] [doi: 10.1186/1472-6947-13-69] [Medline: 23816138]

36. Walsh C, Siegler EL, Cheston E, O'Donnell H, Collins S, Stein D, Informatics Intervention Research Collaboration (I2RC). Provider-to-provider electronic communication in the era of meaningful use: a review of the evidence. J Hosp Med 2013 Oct;8(10):589-597 [FREE Full text] [doi: 10.1002/jhm.2082] [Medline: 24101544]

37. Tenório J, Cohrs F, Pisa I, de Fátima Marin H. Personal health record: a review on current models and experiments. J Health Inform 2013:91-97 [FREE Full text]

38. Moja L, Kwag KH, Lytras T, Bertizzolo L, Brandt L, Pecoraro V, et al. Effectiveness of computerized decision support systems linked to electronic health records: a systematic review and meta-analysis. Am J Public Health 2014 Dec;104(12):e12-e22. [doi: 10.2105/AJPH.2014.302164] [Medline: 25322302]

39. Nguyen L, Bellucci E, Nguyen LT. Electronic health records implementation: an evaluation of information system impact and contingency factors. Int J Med Inform 2014 Nov;83(11):779-796. [doi: 10.1016/j.ijmedinf.2014.06.011] [Medline: $\underline{25085286}$ ]

40. Fiander M, McGowan J, Grad R, Pluye P, Hannes K, Labrecque M, et al. Interventions to increase the use of electronic health information by healthcare practitioners to improve clinical practice and patient outcomes. Cochrane Database Syst Rev 2015 Mar 14(3):CD004749. [doi: 10.1002/14651858.CD004749.pub3] [Medline: 25770311]

\author{
Abbreviations \\ AMSTAR: a measurement tool to assess systematic reviews \\ ATC: anatomical therapeutic chemical \\ CDSS: computerized decision support systems \\ CEN: European Committee for Standardization \\ CHTs: computerized physician handoff tools \\ CPOE: computerized provider order-entry \\ CPR: computer-based patient record \\ EHR: electronic health record
}


EMR: electronic medical record

HAIs: health care-associated infections surveillance systems

HL7: Health level seven

I2: measure of heterogeneity

ICD: International Classification of Disease

ICU: Intensive Care Unit

ISO: International Organization for Standardization

IT: information technology

LOC: level of consciousness

LOS: length of stay

NPV: negative predictive value

OR: odds ratio

PAHO: Pan American Health Organization

PDMS: patient data management system

PPV: positive predictive value

PRISMA: preferred reporting items for systematic reviews and meta-analyses

SpO2: oxygen saturation

SBAR: situation, background, assessment, recommendation

UOP: urine output

WHO: World Health Organization

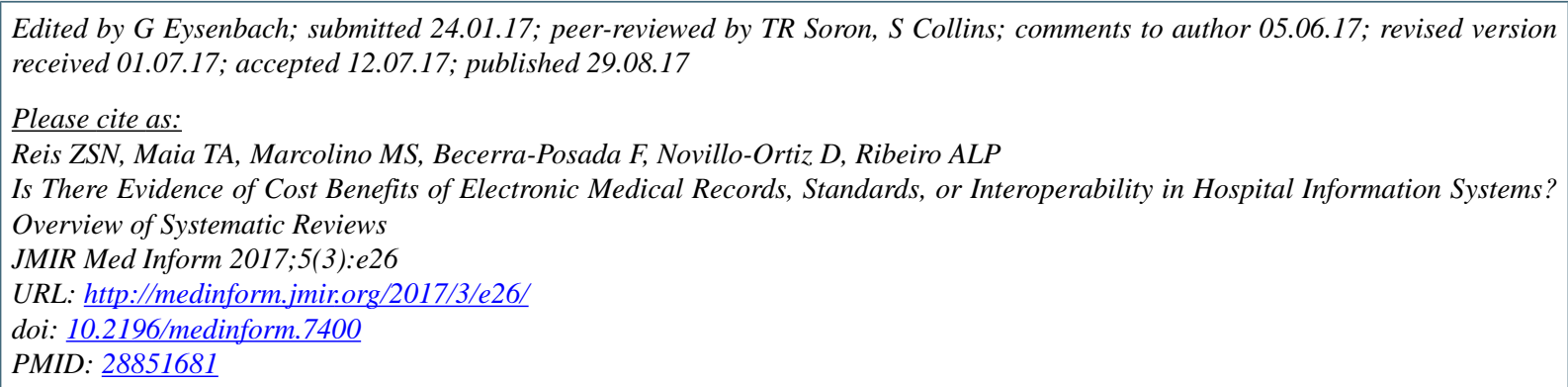

CZZilma Silveira Nogueira Reis, Thais Abreu Maia, Milena Soriano Marcolino, Francisco Becerra-Posada, David Novillo-Ortiz, Antonio Luiz Pinho Ribeiro. Originally published in JMIR Medical Informatics (http://medinform.jmir.org), 29.08.2017. This is an open-access article distributed under the terms of the Creative Commons Attribution License (https://creativecommons.org/licenses/by/4.0/), which permits unrestricted use, distribution, and reproduction in any medium, provided the original work, first published in JMIR Medical Informatics, is properly cited. The complete bibliographic information, a link to the original publication on http://medinform.jmir.org/, as well as this copyright and license information must be included. 